\title{
TURISMO CINEMATOGRÁFICO Y DESARROLLO ECONÓMICO LOCAL. EL FESTIVAL DE CINE DE HUELVA
}

\author{
David Flores Ruiz
}

Universidad de Huelva

\section{RESUMEN}

Analizamos la importancia del fomento del turismo y del desarrollo local a partir de la industria del cine, prestando especial atención al impacto económico local que genera la organización de festivales de cine. Se analiza el caso del Festival Iberoamericano de Cine de Huelva y se concluye que los beneficios económicos de organizar este evento supera a los costes, tal y como se apunta en la bibliografía consultada.

Palabras clave: Turismo cinematográfico; desarrollo económico local; impactos económicos.

Film tourism and local economic development. The Festival of Cinema of Huelva

\section{ABSTRACT}

We analyze the importance of the promotion of the tourism and of the local development from the industry of the cinema. We pay particular attention to the local economic impact of the organization of festivals of cinema. We study the case of the Latin-American Festival of Cinema of Huelva. We conclude that the economic benefits of organizing this event are major that the costs, as the consulted bibliography say.

Keywords: Film Tourism; Local Economic Development, Economic Impacts.

Recibido: 14 de julio de 2014

Devuelto para su revisión: 27 de noviembre de 2014

Aceptado: 16 de diciembre de 2014

Departamento de Economía. Universidad de Huelva. Plaza de la Merced. 21002 HUELVA (España). E-mail: David.flores@dege.uhu.es 


\section{INTRODUCCIÓN}

En las últimas décadas, aunque la demanda turística continúa creciendo, la oferta turística, tanto de empresas como de destinos turísticos, lo hace a mayores tasas, lo cual incide en un importante incremento en los niveles de competencia del sector (Barroso González y Flores Ruiz, 2006). De forma que, para hacer frente a este incremento en los niveles de competencia, los territorios que apuesten por considerar al turismo dentro de sus estrategias de desarrollo local deberán implementar políticas turísticas dirigidas a la creación de una oferta turística atractiva y diversa, la cual resulte realmente competitiva en los mercados turísticos nacionales e internacionales. En este sentido, una de las tipologías de turismos que más está creciendo en los últimos años es el turismo cultural y, dentro de éste, el turismo cinematográfico (Rodríguez et al, 2013), el cual se presenta como uno de las tipologías con mayor potencial de crecimiento a la hora de diseñar la oferta turística de un destino.

Por ello, la presente investigación se centra en el análisis, desde un punto de vista cuantitativo, del impacto económico local que puede generar el turismo cinematográfico, a través de la organización de festivales de cine, como estrategia de desarrollo económico local, aplicado al caso del Festival de Cine Iberoamericano de la ciudad de Huelva.

No obstante, previamente, como marco teórico de la presente investigación, se pone de manifiesto las posibilidades que ofrece el fomento del turismo como estrategia dinamizadora de los procesos de desarrollo económico local para, posteriormente, pasar a conceptuar el turismo cinematográfico, sus tipologías y poner de manifiesto las principales conclusiones a las que llegan algunos de los estudios realizados sobre el impacto económico local que genera la organización de festivales de cine.

Por último, la parte empírica de esta investigación pretende validar, para el caso del Festival de Cine Iberoamericano de Huelva, las conclusiones a las que han llegado otras investigaciones centradas en el análisis del impacto económico local de la organización de eventos culturales, en general y de festivales de cine, en particular.

\section{TURISMO CINEMATOGRÁFICO DE FESTIVALES E IMPACTO ECONÓ- MICO LOCAL}

\subsection{El turismo como estrategia de desarrollo económico local}

El fomento del turismo, por sus especiales características, tal y como vamos a argumentar en el presente epígrafe, se convierte en una importante estrategia a la hora de fomentar procesos de desarrollo económico local. Así, entre las principales características de la actividad turística que pueden contribuir a impulsar y dinamizar estos procesos de desarrollo destacan (Flores Ruiz y Barroso González, 2012):

- Su capacidad para dinamizar y diversificar las economías locales, dado su elevado efecto multiplicador. En este sentido, el desarrollo turístico de un territorio pone a funcionar una parte importante de sus diferentes sectores productivos, tales como: restauración, hotelería, agencias de viajes, transporte, comercio, construcción, ocio, etc. 
- Su capacidad para poner en valor el patrimonio natural y sociocultural de los territorios donde se desarrolla, integrándolos dentro de una estrategia de desarrollo territorial sostenible.

- Su contribución para aumentar la dimensión del mercado local (demanda interna), ya que no debe olvidarse que el desarrollo turístico puede asemejarse con una estrategia de fomento de las exportaciones, al poder aumentar a través de ella la demanda de productos locales.

- Es una actividad intensiva en mano de obra, generadora de importantes puestos de trabajo con volúmenes de inversión no muy elevados.

- La población local ejerce un importante papel en su desarrollo, siendo pieza fundamental a la hora de garantizar el éxito de ese desarrollo turístico. No en vano, el turista, cada vez más, se siente atraído por entrar en contacto con la población local para conocer su cultura.

- Es uno de los principales sectores económicos con mayor potencial de crecimiento, sobre todo en lo que respecta a determinados segmentos de la demanda, entre los que destaca el turismo cultural y, dentro de éste, el cinematográfico (Grande, 2001).

- En los procesos de desarrollo económico local adquieren un importante papel las PYMES y microempresas, las cuales requieren un volumen de inversión no excesivamente altos, siendo gestionadas por la población local. En este sentido, el turismo se convierte en una actividad económica ideal para la inversión en la misma por parte de la población local, ya que la mayor parte de las empresas que participan en esta actividad son PYMES y, por tanto, el volumen de inversión requerido para ello suele ser relativamente bajo.

En definitiva, por sus características específicas, el turismo se configura como una de las actividades dinamizadoras más importantes a tener en cuenta en los procesos de desarrollo económico local. No en vano, en los programas operativos PRODER y PRODER-A, y en las iniciativas comunitarias, LEADER I, LEADER II y LEADER +, los proyectos a los que se han dirigido un mayor volumen de financiación han sido proyectos relacionados, de una forma u otra, con la actividad turística, tales como: infraestructuras turísticas, creación de empresas turísticas, formación de los recursos humanos en temas relacionados con el turismo, promoción turística, etc. (Consejería de Agricultura y Pesca, 2003a, 2003b).

\subsection{Turismo cinematográfico: conceptualización y tipologías}

Cabe reseñar la importancia que, cada vez más, está experimentando el turismo cultural para la gestión de los destinos turísticos. Esta importancia, según Rodríguez et al (2013), descansa en los siguientes aspectos:

- El importante crecimiento cuantitativo que viene experimentando este tipo de demanda como consecuencia de las actuales tendencias sociales y económicas del turismo (Grande, 2001), lo cual hace que dicho crecimiento sea superior a la media del sector. 
- La importancia que, desde un punto de vista cualitativo, adquiere esta tipología de turismo como elemento de desestacionalización, especial y temporal, de una oferta y demanda turística como la española, basada, en un gran porcentaje, en el turismo de sol y playa. No en vano, tal y como recoge Martínez Fraile, España es el cuarto destino cultural europeo y ha conseguido decantar casi un 5\% de los turistas de sol y playa hacia propuestas culturales ${ }^{1}$.

- La tendencia creciente, por parte del turista, a una búsqueda de nuevas satisfacciones en respuestas a expectativas de ocio y esparcimiento, demandando una combinación de vacaciones, turismo y enriquecimiento cultural.

- La diversificación, cada vez más, de las motivaciones del turista. De tal forma, que actualmente es difícil satisfacer a todos los turistas, atraídos por el turismo cultural, ofertándole un sólo producto turístico. En este contexto, surgen y adquieren importancia, diferentes tipologías de turismos específicos, pertenecientes al turismo cultural, tales como: turismo arquitectónico, museístico, gastronómico, idiomático, religioso, musical, cinematográfico, etc.

En definitiva, todos estos aspectos hacen que el turismo cultural vaya adquiriendo especial importancia en las últimas décadas, tanto desde el punto de vista cuantitativo como cualitativo, a la hora de aumentar la calidad y la competitividad de los destinos turísticos. En este sentido, se manifiesta Richards (1996) al afirmar que el turismo cultural es uno de los segmentos que más ha crecido en los últimos años, siendo los festivales un importante atractivo turístico, ya que permiten cubrir simultáneamente las necesidades de ocio y de cultura de los individuos (Yeoman et al., 2004).

No obstante, podría decirse que, aunque el turismo cinematográfico se suele clasificar como una subtipología de turismo cultural (McKercher y Cross, 2002; Hudson y Brent, 2006), hay autores que no opinan así, ya que consideran que el cine no siempre muestra aspectos reales y, por tanto, culturales. De hecho, hay obras que muestran aspectos irreales que nada tienen que ver con la cultura y la forma de vivir de una determinada sociedad. Por este motivo, este grupo de autores minoritarios incluyen al turismo cinematográfico dentro de la categoría «otros». En nuestro estudio, consideraremos al turismo cinematográfico como una tipología que se incluye dentro del turismo cultural, tal y como es considerada por la mayoría de autores que lo estudian (Domínguez Azcue, 2014).

A pesar de las relaciones históricas que mantiene la actividad turística con el cine ${ }^{2}$, el turismo cinematográfico, al ser una tipología que comienza a adquirir cierta importancia en las últimas décadas, ha sido definido y caracterizado por pocos autores. Uno de los que sí lo ha hecho es Beeton (2005), el cual hace referencia al «turismo inducido por el cine» como el turismo realizado en un lugar como consecuencia del éxito de una película que fue ambientada o bien rodada en una región concreta. Por su parte, Evans (1997)

1 Intervención realizada por el entonces Secretario de Turismo con motivo de la promoción conjunta con carácter cultural realizada por el organismo que representaba y la Fundación Gala-Dalí.

2 Tal y como reconoce Del Rey-Reguillo (2007), el cine y el turismo han desarrollado su historia en paralelo, llegando a convertirse en dos de las industrias más potentes del mundo contemporáneo, surgiendo ambas en el S. XIX, con una diferencia de medio siglo. 
considera que comprende las visitas realizadas por turistas a un destino o atracción como resultado de que dicho destino apareciera en televisión, vídeo o pantalla de cine.

De esta forma, puede decirse que, debido a su enorme poder de seducción sobre la imaginación del espectador, el cine comienza a ser considerado como un agente de primer orden para inducir la mirada del turista y, por tanto, condicionar la elección de los destinos (Del Rey-Reguillo, 2007). No en vano, el cine, a través de la narración y reproducción de un contexto y realidad diferenciada, nos va a aportar una manera enriquecida de entender cómo es nuestro entorno y que significa en nuestro contexto social, cultural y económico, permitiendo mostrar no sólo el presente, sino el futuro (Martínez Puche, 2008: 147). De hecho, tal y como recoge Gámir y Valés (2007: 185), son numerosos los ejemplos de películas que contribuyen al incremento de visitas turísticas, mostrando el interés de los espectadores-turistas por vincular los lugares observados por el cine y los que contemplan en sus viajes.

Estas imágenes de un territorio percibidas a través de un producto audiovisual pueden influir en el comportamiento de los consumidores turísticos. Por ello, se debe actuar de tal manera que la imagen que se proyecta del territorio en el cine constituya un elemento diferenciador de la competencia y permita a ésta posicionarse favorablemente en la mente de los potenciales turistas; sobre todo si tenemos en cuenta la etapa de crecimiento que está experimentando el turismo cinematográfico como consecuencia del crecimiento de la industria de entretenimiento y por el aumento del número de viajes internacionales (Rodríguez Campos, y Fraiz Brea, 2011:52).

Por todo ello, Rodríguez et al (2013:264) consideran que la necesidad de innovar, de ser creativos y diferenciarse ha llevado a destinos turísticos de todo el mundo a ser pioneros en la utilización del cine tanto para promocionarse como para diversificar su oferta. Y, como consecuencia de esto, en España, en las últimas décadas, han ido apareciendo diversos estudios empíricos que corroboran esta tesis (Sandoval Martín, 1998; Rodríguez Campos y Fráiz Brea, 2010; Rodríguez Campos y Fráiz Brea, 2011).

Una vez valorada la importancia del cine como elemento de atracción turística, las autoridades y empresas de turismo del destino, para conseguir atraer rodajes, desarrollan las Film Commission, como oficinas públicas sin ánimo de lucro cuyo objetivo es atraer y gestionar rodajes audiovisuales. Así, por ejemplo, en Andalucía, en 1988, se crea Andalucía Film Commission con el fin de promocionar la comunidad como lugar de rodaje y apoyo a las empresas y profesionales de la industria audiovisual en la logística de sus producciones. Para ello, proporciona información sobre posibles localizaciones y coordina la industria audiovisual y las compañías de producción con las diferentes administraciones públicas. Con ello, se pretende que el cine contribuya al fomento del turismo y la economía del territorio andaluz. Para llevar a cabo su labor, actualmente tiene suscrito acuerdos con algunas provincias andaluzas -entre las que se encuentra la ciudad de Huelva- con el fin de crear la red de ciudades de cine $^{3}$.

En definitiva, considerando a los anteriores autores, el turismo cinematográfico podría ser definido como aquel tipo de turismo que es generado como consecuencia del rodaje

3 Para una mayor información sobre el caso de Andalucía Film Commision consultar: http://www.andaluciafilm.com/index.php/inicio 
de largometrajes o de la organización de festivales de cine, pudiendo adoptar diferentes formas en función al tipo de localización que se proyecta en la película o serie, y de cómo ésta ha afectado en la motivación del individuo. Beeton (2005) hace un esfuerzo por clasificar las diferentes formas en que se puede manifestar el turismo cinematográfico, las cuales quedan recogidas en el siguiente cuadro.

\section{Cuadro 1}

\section{TIPOS Y CARACTERÍSTICAS DEL TURISMO CINEMATOGRÁFICO}

\begin{tabular}{|c|c|c|}
\hline TIPO & CARACTERÍSTICAS & EJEMPLOS \\
\hline \multicolumn{3}{|c|}{ ESCENARIOS REALES (ON LOCTION) } \\
\hline $\begin{array}{l}\text { Turismo cinematográfi- } \\
\text { co como elemento prin- } \\
\text { cipal que motiva el viaje }\end{array}$ & $\begin{array}{l}\text { El lugar de rodaje, el escenario real y } \\
\text { natural de la película es una atracción } \\
\text { en sí misma lo suficientemente fuerte } \\
\text { como para motivar el viaje }\end{array}$ & Isla de Mull (Balamory) \\
\hline $\begin{array}{l}\text { El turismo cinematográ- } \\
\text { fico como parte de unas } \\
\text { vacaciones }\end{array}$ & $\begin{array}{l}\text { Realizar visitas a lugares de rodaje de } \\
\text { películas como una actividad dentro } \\
\text { de unas vacaciones más amplias }\end{array}$ & \\
\hline $\begin{array}{l}\text { El turismo cinematográ- } \\
\text { fico de peregrinaje }\end{array}$ & $\begin{array}{l}\text { Visitar los lugares de rodajes de pelí- } \\
\text { culas como una forma de «prestar un } \\
\text { homenaje» a la película }\end{array}$ & $\begin{array}{l}\text { Doune Castle (Monty } \\
\text { Python) } \\
\text { Lugares de rodaje (El Señor } \\
\text { de Los Anillos) }\end{array}$ \\
\hline $\begin{array}{l}\text { El turismo cinematográ- } \\
\text { fico de celebridades }\end{array}$ & $\begin{array}{l}\text { Los hogares de las celebridades; lu- } \\
\text { gares de rodaje de películas que han } \\
\text { tomado el estatus de celebridades }\end{array}$ & Cass de Hollywood \\
\hline $\begin{array}{l}\text { El turismo cinematográ- } \\
\text { fico nostálgico }\end{array}$ & $\begin{array}{l}\text { Visitas a los lugares de rodaje de pe- } \\
\text { lículasque representan otras eras }\end{array}$ & $\begin{array}{l}\text { The Andy Griffith Show } \\
\text { (años 50) } \\
\text { Heartbeat (años 60) }\end{array}$ \\
\hline \multicolumn{3}{|l|}{ COMERCIAL } \\
\hline $\begin{array}{l}\text { Atracciones turísticas de } \\
\text { turismo cinematográfico } \\
\text { construidas }\end{array}$ & $\begin{array}{l}\text { Una atracción construidas después del } \\
\text { rodaje de una película exclusivamente } \\
\text { para atraer y servir a los turistas }\end{array}$ & La experiencia Heartbeat \\
\hline Tours de cine/películas & $\begin{array}{l}\text { Rutas desarrolladas para varias loca- } \\
\text { lidades de rodaje de películas }\end{array}$ & $\begin{array}{l}\text { On localitations tours en } \\
\text { Nueva York }\end{array}$ \\
\hline $\begin{array}{l}\text { Rutas guiadas por el es- } \\
\text { cenario del rodaje }\end{array}$ & $\begin{array}{l}\text { Rutas de lugares específicos, con fre- } \\
\text { cuencia en zonas de propiedad privada }\end{array}$ & Hobbiton \\
\hline \multicolumn{3}{|c|}{ IDENTIDADES EQUIVOCADAS } \\
\hline $\begin{array}{l}\text { Turismo cinematográfi- } \\
\text { co donde solamente se } \\
\text { cree que el rodaje ha te- } \\
\text { nido lugar }\end{array}$ & $\begin{array}{l}\text { Películas y series de televisión que } \\
\text { son rodadas en un lugar que es crea- } \\
\text { do para parecerse a otro; con fre- } \\
\text { cuencia en otros países por razones } \\
\text { financieras }\end{array}$ & $\begin{array}{l}\text { Deliverance se rodó en una } \\
\text { Claybum aunque está inspi- } \\
\text { rada en Appalachia }\end{array}$ \\
\hline $\begin{array}{l}\text { Turismo cinematográfico } \\
\text { en lugares en los que la } \\
\text { película se inspira pero } \\
\text { no se realiza el rodaje }\end{array}$ & $\begin{array}{l}\text { La película ha aumentado el interés } \\
\text { en un país, región o lugar en particu- } \\
\text { lar, en el que se basa la historia pero } \\
\text { no se rodó realmente }\end{array}$ & $\begin{array}{l}\text { Braveheart se rodó en } \mathrm{Ir}- \\
\text { landa aunque se cree que se } \\
\text { rodó en Escocia }\end{array}$ \\
\hline
\end{tabular}




\begin{tabular}{|c|c|c|}
\hline \multicolumn{3}{|c|}{ ESCENARIOS CREADOS (OFF LOCATION) } \\
\hline $\begin{array}{l}\text { Rutas por los estudios de } \\
\text { cine }\end{array}$ & $\begin{array}{l}\text { Rutas de la industria por el trabajo } \\
\text { de estudios cinematográficos, en los } \\
\text { que el proceso real de rodaje puede } \\
\text { ser visto }\end{array}$ & Estudios Paramount \\
\hline $\begin{array}{l}\text { Parques temáticos en los } \\
\text { estudios de cine }\end{array}$ & $\begin{array}{l}\text { Generalmente relacionados con un } \\
\text { estudio, se trata de construcciones } \\
\text { realizadas específicamente para el tu- } \\
\text { rismo sin que producciones cinema- } \\
\text { tográficas estén teniendo lugar }\end{array}$ & Estudios Universal \\
\hline \multicolumn{3}{|l|}{ EVENTOS ONE-OFF } \\
\hline Estrenos De películas & $\begin{array}{l}\text { En particular aquellos eventos que } \\
\text { tienen lugar fuera de lugares tradicio- } \\
\text { nales como es el caso de Holywood }\end{array}$ & $\begin{array}{l}\text { El Señor de los Anillos: el re- } \\
\text { torno del rey (Nueva Zelanda) } \\
\text { Misión Imposible II (Sydney) }\end{array}$ \\
\hline Festivales de cine & $\begin{array}{l}\text { Numerosas ciudades cuentan con } \\
\text { festivales de cine que atraen al even- } \\
\text { to aficionados y fanáticos del cine }\end{array}$ & $\begin{array}{l}\text { Cannes } \\
\text { Edinburgo }\end{array}$ \\
\hline $\begin{array}{l}\text { Programas de televisión } \\
\text { sobre viajes }\end{array}$ & $\begin{array}{l}\text { Son los sucesores de las guías de via- } \\
\text { jes tradicionales y los documentos de } \\
\text { viajes escritos }\end{array}$ & $\begin{array}{l}\text { Getaway } \\
\text { Pilot Guies }\end{array}$ \\
\hline $\begin{array}{l}\text { Programas gastronómi- } \\
\text { cos }\end{array}$ & $\begin{array}{l}\text { Numerosos espectáculos de cocina } \\
\text { llevan al espectador a varios lugares } \\
\text { alrededor del mundo }\end{array}$ & Cook's Tour \\
\hline
\end{tabular}

Fuente: Beeton (2005).

Según se desprende del Cuadro 1, podría decirse que, a pesar de la motivación general que mueve al turista cinematográfico por el cine, esta tipología turística no atiende a un grupo homogéneo de individuos, ya que cada película y serie tiene su grupo específico de turistas con características propias. Por tanto, podría decirse que el turismo cinematográfico comprende, a su vez, una gran variedad de subtipologías turísticas. En nuestro estudio nos centramos en el análisis de la demanda de una de ellas, la cual hace referencia al turismo cinematográfico de festivales.

A pesar de toda esta variedad de tipologías de turismo cinematográfico, Domínguez Azcue (2014) hace un esfuerzo por sintetizarlas, conformando los siguientes tres grandes grupos de tipologías: a) turismo cinematográfico en escenarios reales: son lugares del entorno real que los turistas deciden visitar después de haberlos visto, o creer haberlos visionado, en la pantalla; b) turismo cinematográfico en escenario creados: son localizaciones artificiales, creadas específicamente para poder rodar un película o con propósitos turísticos, como los parques de atracciones cinematográficos. Otra tipología considerada aquí son los estrenos de películas o festivales de cine, que, tal y como reconocen Beeton (2005) y Roesch (2009), pueden ocasionar un incremento de turistas y visitantes en el destino; y c) turismo cinematográfico como turismo de negocios, el cual se da cuando un destino llega a un acuerdo con una productora para rodar una película en el mismo y que, de esta forma, todos los trabajadores de la productora (actores, guionistas, directores, maquilladores, peluqueros, etc.), pasen a ser turistas en el propio destino. 
En los siguientes apartados profundizamos en el análisis de una de estas tipologías de turismo cinematográfico, nos referimos al turismo cinematográfico de festivales, prestando especial atención a las repercusiones económicas que tiene para los territorios donde se desarrollan. Posteriormente, se analiza el impacto económico que general el Festival de cine Iberoamericano para la ciudad de Huelva.

\subsection{El turismo cinematográfico de festivales}

Tal y como recoge Elizagarate (2008: 140), la política cultural que vienen impulsando las ciudades con el desarrollo de actividades en el marco de las artes escénicas, como la música, el teatro, los museos y el cine son en la actualidad no sólo un factor de atracción de turismo urbano sino una demanda de calidad de vida que tiene una gran capacidad para generar empleo directo y desarrollo económico local.

En esta línea, la denominada «civilización del ocio» y, por tanto, la demanda de cultura, ha impulsado el desarrollo de una red de infraestructuras culturales con gran impacto en la regeneración urbana de la ciudad, que crean centralidad y son motores del desarrollo económico por el impacto de las inversiones y el gasto generado por los visitantes ${ }^{4}$.

Así por ejemplo, el Palacio de Congresos Kursaal de San Sebastián tiene una facturación anual de 3,1 millones de euros y atrajo a 2,6 millones de visitantes en el período 1999-2005, albergándose en él toda la oferta cultural de la ciudad, cuyos eventos más importantes son el Festival Internacional de Cine y el Festival Internacional de Cine Publicitario ${ }^{5}$.

Por tanto, siguiendo a García y Alburquerque (2003), podríamos decir que estas infraestructuras que potencian el turismo de festivales (tipología que se encuentra dentro del turismo cinematográfico, tal y como se viene recogiendo en el presente trabajo), constituyen un buen ejemplo de oferta turística complementaria, la cual, en algunos casos, puede llegar a convertirse en el principal motivo de la visita al destino turístico, tal y como es el caso del Festival Internacional de Cine de San Sebastián.

Así, la celebración de un festival de cine suele ser valorado por los consumidores de este tipo de eventos culturales como el principal motivo, o un motivo complementario, en la elección de una determinada ciudad como destino turístico (García y Alburquerque, 2003), representando, al mismo tiempo, un punto de encuentro donde los directores, actores, productores, guionistas,... pueden dar a conocer su trabajo al gran público fuera de los circuitos comerciales estandarizados.

En la práctica pueden suponer la entrada de flujos de ingresos provenientes de los asistentes a este tipo de eventos, con una incidencia directa e indirecta sobre el tejido empresarial local, tanto en la generación de riqueza como de puestos de trabajo. Por ello, se pueden llegar a convertir en un recurso importante para el desarrollo económico local; y, al mismo tiempo, contribuir a paliar el efecto estacional turístico asociado a distintas localidades.

4 Un ejemplo de cómo la economía de la cultura influye en la regeneración urbana de las ciudades, así como en el desarrollo socioeconómico de las mismas, lo encontramos en la ciudad de Bilbao, la cual, a partir de la construcción del Museo Guggenheim, que se convierte en imagen de marca de la ciudad, pasa de ser considerada una ciudad industrial en declive a convertirse en la nueva «Meca del Urbanismo» (Rodríguez, 2002 y Guggenhein Bilbao, 2003).

5 http://www.kursaal.com.es/ página consultada en 3 de diciembre de 2014. 
A su vez, tal y como afirman Devesa y Herrero (2008), desde un punta de vista cualitativo, los festivales tienen un valor cultural fundamental, ya que permiten el acceso a las artes, aumentan la oferta cultural de las ciudades, refuerzan la identidad de la comunidad o mejoran el bienestar de los ciudadanos. Asimismo se convierten en un escaparate turístico para la ciudad que mejora la imagen de la misma, tanto para los turistas nacionales como extranjeros. A título ilustrativo podemos citar el importante impacto positivo que tradicionalmente han reportado festivales celebrados en los Ángeles (California), con la entrega de los Oscar, Cannes en Francia, Venecia o la Berlinae en Alemania. Incluso puede constituirse en una de los pilares sobre la que se define la política cultural de la localidad.

En definitiva, dada la importancia que, tanto para la población local como para el turista, adquiere la organización de este tipo de eventos, en los últimos años comienzan a aparecer en la literatura científica múltiples trabajos de investigación que tienen como principal objetivo determinar el impacto económico local que generan (Frey, 2004; Asuaga et al, 2011; Capaul, 1988; Herrero et al, 2011; Devesa, 2006; Devesa et al, 2012; García et al, 2003; Gilbson, et al, 2010; O’Hagan, 1989; Perles, 2006; Scotiish Tourist Borrad, 1996; Shellard, 2007; Tyrrel y Ismael, 2005; entre otros). En este sentido, en el siguiente epígrafe comentamos las principales conclusiones a las que se llegan en algunas de estas investigaciones, así como la metodología en la que se basan, con objeto de poder contrastar estas conclusiones para el caso del Festival de Cine Iberoamericano de Huelva.

\subsection{El impacto económico local del turismo cinematográfico de festivales}

Como manifiesta Espinal (2006) en la sociedad actual la cultura ha pasado de ser un placer escaso a convertirse en un exigencia de muchos ciudadanos, de ser accesoria a ser esencial para vivir, de ser un producto para la distracción a ser uno de los consumos más habituales, de ser minoritaria y elitista a ser consumo de masa, apareciendo el concepto de la «sociedad del ocio» y el desarrollo de la denominada como Economía de la Cultura.

En este sentido, a la hora de valorar todos estos aspectos culturales, Thorby (2001) considera que en cualquier modelo que se aplique para la determinación del valor de todo evento cultural, debe de tenerse en cuenta la existencia de bienes privados excluibles, bienes públicos y las externalidades.

Así, centrándonos en los festivales de cine, podría decirse que éstos representan muchas realidades distintas, que pueden ser objeto de distintas valoraciones. De forma que, para los directores de cine, actores, fotógrafos, productores, cámaras,..., suponen un instrumento a través del cual pueden dar a conocer su obra y su trabajo; para las personas que directamente intervienen en el festival suponen desarrollar una actividad retribuida; para los comerciantes de la zona, hoteleros, restauradores,..., supone un incremento de sus ventas durante el período de tiempo en el cual se está desarrollando esta actividad cultural. Por lo tanto, el valor económico depende tanto del contenido patrimonial como del conjunto de flujos de bienes y servicios que se generan.

Un modelo que ha sido utilizado frecuentemente para el estudio del impacto económico de los eventos culturales sobre el territorio es el método de los efectos (Devesa, 2006; Herrero et al, 2006; Devesa y Herrero, 2008; Devesa et al, 2012, entre otros), el cual se aplica en el presente trabajo para determinar el impacto económico del Festival de 
Cine Iberoamericano en la ciudad de Huelva. Este modelo permite determinar los flujos de ingresos, relacionados con un evento cultural, como es el caso de los festivales de cine, y con ello determinar los efectos del mismo sobre un territorio. Para ello, se define la existencia de tres tipos de impactos o efectos, como son: los directos, los indirectos y los inducidos.

- Los efectos directos están determinados por los gastos llevados a cabo por la organización del evento cultural. Dentro de ellos pueden encontrarse los destinados al funcionamiento del festival, tales como gastos de personal oficina y administración, asesoramiento legal y económico, financieros o impuestos. Otras partidas que también pueden resultar interesantes son las derivadas de los propios actos de la organización, tales como los de dirección, premios, invitados, programación, producción, comunicación, publicidad, seguros y otros gastos amortizables. Cuestión importante es la de delimitar de forma directa los gastos que sean imputables al propio evento que se desarrolla.

- Los efectos indirectos vienen definidos por los ingresos provenientes de los consumidores de este tipo de eventos. Estarían constituidos por los provenientes de la venta de entradas, merchandising, gasto realizado por los visitantes foráneos en la ciudad (hotelería, restauración, transporte, etc.), etc.

- Los efectos inducidos, tales como la mejora de la calidad de vida de los ciudadanos, el incremento del capital humano del territorio donde se celebra el evento, repercusiones económicas sobre el resto de la economía local o regional, etc. En este último caso es frecuente recurrir para su cálculo a los multiplicadores de la economía, provenientes de las tablas input-output de Leontief.

El mundo del cine, en general, y el específico de los festivales, en particular, han sido tratados por numerosos autores, poniendo de manifiesto la potencialidad del séptimo arte sobre el desarrollo económico local. Así, los autores Devesa y Herrero (2008) aplican la metodología de los estudios de impacto económico a la $46^{\text {a }}$ Edición de la Semana Internacional de Cine de Valladolid que se celebró entre el 25 de octubre y el 3 de noviembre de 2001. Entre sus principales conclusiones se encuentran: a) que los ingresos para la economía local durante la celebración del mismo ascendieron a 2,3 millones de euros y permitió crear 39 puestos de trabajo, entre empleos directos, indirectos e inducidos; b) que la realización del festival afectó básicamente al sector turístico de la ciudad; y c) condujo a una circulación activa de fondos entre agentes públicos y privados vinculados al territorio.

Al mismo tiempo, ambos autores ponen de manifiesto cómo a través del festival se mejoró la imagen de la ciudad, con las implicaciones que pudiesen derivarse a corto y medio plazo sobre el turismo de la misma. Igualmente destacan la capacidad que tienen este tipo de eventos para diversificar la oferta cultural, preservar la cultura local, mejorar el bienestar de los ciudadanos o aumentar la cohesión social. En definitiva, podría concluirse cómo este festival contribuye al dinamizar el desarrollo local de Valladolid, no solamente en lo que a los aspectos económicos y cuantitativos respecta, sino también en temas sociales, culturales, de mejora de la calidad de vida de la población local, etc. 
Por su parte, los autores Herrero et al (2006) realizan un estudio con ocasión de la elección de la ciudad de Salamanca como capital europea de la cultura. La metodología que emplearon fue también la del impacto económico y entre sus principales conclusiones caben destacar: a) que este acontecimiento generó en Castilla y León 556,1 millones de euros y otros 247,2 millones en el resto de España; b) que la distribución sectorial del impacto económico indica que un $65,7 \%$ de las repercusiones económicas de Salamanca 2002 provienen de dicho evento, y el 34,3\% restante estaban relacionadas con el impacto económico de la dotación de las nuevas infraestructuras culturales y equipamiento turístico; y c) que el impacto del gasto cultural en la Comunidad Autónoma es de mayor dimensión que el impacto del gasto dotacional.

Por tanto, los resultados a los que llegan todos estos autores ponen de manifiesto el importante potencial que tiene la organización de este tipo de eventos como estrategia de desarrollo económico local; pudiendo llegar a ser mayor que las meras inversiones en infraestructura y obra civil. Por lo que aconsejan la necesidad de incluir la dimensión cultural endógena en el planteamiento de las políticas de desarrollo económico local.

De esta manera y, aun tomando una precaución razonable por la comparación de aplicaciones diferentes, por el objeto y, probablemente, por la metodología, podemos mencionar como elemento de comparación de estos resultados, la estimación de impacto económico de las actividades culturales y la capacidad de atracción turística del Museo Guggenheim de Bilbao, que en el año 2002 se estima en un volumen de 162,3 millones de euros, y de 816,7 millones de euros si acumulamos el impacto durante los siete primeros años de vida del museo (Guggenheum Bilbao, 2003). En consecuencia, atendiendo solamente al impacto del gasto estrictamente cultural de Salamanca 2002, el efecto ha sido 3,2 veces superior a lo generado por el Museo Guggenheim en el mismo año, o el $64 \%$ de lo acumulado en los años de existencia de este museo.

Por otra parte, en otro estudio, Devesa et al (2012) también aplican la metodología de impactos económicos al Festival Internacional de Cine de Valdivia (FICV). Como conclusiones de este estudio cabe destacar las siguientes: a) el festival generó en 2008 unos ingresos en la economía regional que se cifraron en 352,9 millones de pesos; b) muestra una especial incidencia sobre el sector turístico de la ciudad, mediante la generación de una circulación activa de fondos entre los distintos agentes locales, públicos y privados; c) evidencia la existencia de un gasto superior de los espectadores locales; d) los efectos económicos territoriales de este tipo de eventos afectan fundamentalmente a la ciudad que acoge al festival y se va diluyendo según nos alejamos geográficamente del núcleo fundamental de gasto; y e) entre los aspectos mejor valorados por los encuestados se encuentran: sus repercusiones culturales, la menor importancia relativa a los posibles beneficios en la atracción de turismo, así como las mejoras sociales o de impacto económico.

En definitiva, como conclusión a todos estos estudios, podría decirse que los festivales, en general, y los de cine, en particular, adquieren un papel fundamental a la hora de diversificar la oferta cultural de las ciudades, de preservar la cultura local, de mejorar el bienestar de los ciudadanos y de aumentar la cohesión social, contribuyendo también, al desarrollo económico local.

No en vano, desde un punto de vista puramente económico y cuantitativo, los estudios del impacto económico, realizados a través del método de los efectos, ponen de manifiesto 
el importante potencial que tienen estos eventos a la hora de generar flujos económicos en los territorios donde se desarrollan, superando los ingresos a los gastos que puedan ocasionar la organización de los mismos. En el siguiente apartado se pretende contrastar esta hipótesis para el caso del Festival de Cine Iberoamericano de Huelva.

\section{EL IMPACTO ECONÓMICO DEL FESTIVAL DE CINE IBEROAMERICANO}

El Festival Iberoamericano de Huelva se viene realizando de forma continuada desde 1974, y está centrado en el cine desarrollado en países americanos y europeos de lengua española y portuguesa. Desde sus orígenes, se ha caracterizado por convertirse en la puerta de entrada al circuito nacional y europeo de la industria cinematográfica latinoamericana, aprovechando las relaciones que mantiene históricamente la ciudad con estos países. Actualmente es uno de los eventos cinematográficos con mayor arraigo y proyección internacional de los que se celebran en España.

\subsection{Metodología}

Para calcular el impacto económico local que genera el Festival de Cine Iberoamericano en la ciudad de Huelva se recurrió al método de los efectos, siendo las fuentes principales de información las siguientes:

- Una encuesta estructurada que se le paso a los asistentes al Festival.

- La información proporcionada por la organización del Festival, fundamentalmente, la proveniente del presupuesto de esa $36^{\mathrm{a}}$ edición, desglosado por partidas.

El cuestionario se aplicó en tres puntos estratégicos de la ciudad como lo son: Aqualón, Casa de Colón y en Gran Teatro. Dichos lugares fueron los escenarios para la presentación de las muestras de cine iberoamericano, teniendo una gran concentración de espectadores a lo largo de su semana de duración.

En lo que respecta a la encuesta estructurada, ésta se realizó de la siguiente manera:

- Se pasó un cuestionario entre los asistentes de evento cinematográfico totalmente voluntaria, anónima y confidencial, que fue rellenada por todas aquellas personas que quisieron colaborar. El número de encuestas válidas que se obtuvo fue de 732.

- El cuestionario se elaboró tomando como base el elaborado para el análisis de los impactos del Festival Internacional de Cine de Valladolid, llevado a cabo el año 2001, ya que el objetivo de ambos estudios fueron prácticamente coincidentes (Devesa, 2006). A demás, ello puede permitir, en estudios posteriores, realizar determinadas comparaciones entre ambos festivales, lo cual puede enriquecer sustancialmente los resultados de esta investigación.

- Se iniciaron la cumplimentación del cuestionario (trabajo de campo) el domingo 14 y finalizó el 20 de noviembre de 2010, con la ayuda y colaboración de alumnos/as de la Universidad de Huelva, previamente formados en el proceso de encuestación. 
- Los datos se trataron con el programa estadístico del SPSS, comparando siempre el perfil del asistente al festival procedente de fuera (visitante), respecto al que reside en la propia ciudad, con objeto de poner de manifiesto los ingresos económicos que proceden de las visitas que se hacen a la ciudad de Huelva, motivadas, fundamentalmente, por la asistencia al festival (turismo cinematográfico de festival).

En la elaboración del cuestionario se decidió que las preguntas fueran cerradas (cuestionario estructurado). Se estructuró en 21 preguntas que permitió recoger la siguiente información:

- Datos generales (sexo, edad, residencia habitual, ocupación, estado civil, nivel de estudios finalizado e ingreso mensual neto aproximado)

- Tipo de entrada que habían adquirido

- Residencia habitual

- En caso de ser foráneo, el motivo del viaje a la ciudad.

- Período de permanencia en la ciudad

- Gasto medio diario

- Su asistencia al festival en anteriores ediciones

- Número de películas que prevé el encuestado que va a visualizar.

- Motivación para asistir al festival.

- Su asistencia a otros festivales nacionales o internacionales

- Frecuencia con la que asiste al cine

- La importancia que le da al festival sobre aspectos tales como la posibilidad de ver cine alternativo, la importancia sobre la economía de la ciudad, la atracción sobre el turismo, las oportunidades que se pueden generar para nuevos realizadores, la promoción del cine iberoamericano, el sentimiento de orgullo local, la imagen de la ciudad, etc.

- Valoración global de la presente edición.

Se realizaron 732 encuestas válidas, con un error muestral de $\pm 5 \%$ para un nivel de confianza del $95 \%$. En el siguiente cuadro se recoge una ficha con los todos estos datos de la encuesta.

\section{Cuadro 2 \\ FICHA TÉCNICA}

\begin{tabular}{|l|l|}
\hline Universo & Asistentes a la 36 edición: 27.901 \\
\hline Ámbito geográfico & Ciudad de Huelva \\
\hline Tamaño muestral & 732 \\
\hline Error muestral & $5 \%$ \\
\hline Nivel de confianza & $95 \%$ \\
\hline Fecha de trabajo de campo & $14-20$ de noviembre del 2010 \\
\hline
\end{tabular}

Fuente: Elaboración Propia.

El objetivo de la investigación y, por tanto, el diseño de la encuesta, estaba dirigido a analizar el impacto económico que la organización de este festival de cine genera en la ciudad. 
Para calcular las repercusiones económicas del Festival de Cine Iberoamericano se sigue la aplicación de la metodología de estudios de impactos, para lo cual, tal y como ha quedado recogido en epígrafes anteriores, se calculan tres tipos de efectos económicos: efectos directos (gastos realizados por la organización del Festival de Cine en la ciudad de Huelva), efectos indirectos (gastos de los espectadores relacionados con su asistencia, los cuales son calculados mediante la encuesta) y efectos inducidos (calculados a través de multiplicadores derivados de la Tabla Input-output de la economía andaluza, correspondientes a 1995).

\subsection{Análisis del impacto económico del Festival}

A continuación se recoge el análisis y los resultados que se obtuvieron a la hora de calcular los tres efectos económicos del Festival de Cine Iberoamericano sobre la ciudad de Huelva.

\section{Efecto directo}

Debemos señalar que el Festival de Cine Iberoamericano gastó en 2010 un total de 682.067 $€$, de los cuales la mayor parte quedó en la ciudad de Huelva, repercutiendo, por tanto, en la economía local. Las partidas en las que se desglosa esta cifra aparecen recogidas en el Cuadro 3.

\section{Cuadro 3}

\section{GASTOS DEL FESTIVAL DE CINE IBEROAMERICANO}

\begin{tabular}{|l|c|c|}
\hline \multicolumn{1}{|c|}{ Partidas } & Gasto total & Gasto total en Huelva \\
\hline Personal & 82.674 & 82.674 \\
\hline Invitados & 109.876 & 109.876 \\
\hline Programa & 153.626 & 153.626 \\
\hline Administración & 159.973 & 159.973 \\
\hline Premios & 69.341 & 0 \\
\hline Impuestos & 26.978 & 0 \\
\hline Otros: Comunicación y publicidad & 79.599 & 79.599 \\
\hline TOTAL & $\mathbf{6 8 2 . 0 6 7}$ & $\mathbf{5 8 5 . 7 4 8}$ \\
\hline
\end{tabular}

Fuente: Festival de Cine Iberoamericano y elaboración propia.

Por tanto, consideramos que, de los $682.067 €$ que se gastó la organización del Festival de Cine Iberoamericano en 2010, $585.748 €$ quedaron en la ciudad de Huelva, ya que tanto los premios como los impuestos repercuten, fundamentalmente, en otros territorios. A demás, parte de otras de las partidas consideradas también han podido ir dirigidas fuera de la economía local. No obstante, por desconocerse este dato, se estima que todo el montante queda en la economía onubense.

Así, del dinero gastado en la economía local, la partida más importante es la relacionada con los diferentes gastos de administración (27,31\%), seguidos de la programación $(26,22 \%)$ y de los gastos de invitados (18,75\%). 


\section{Efecto indirecto}

El objetivo principal es «medir las rentas generadas por este evento cinematográfico sobre la economía de la ciudad» de Huelva. Para lograr este objetivo se formuló un cuestionario. Este cuestionario se aplicó en 3 puntos estratégicos de la ciudad: Aqualón, Casa Colón y Gran Teatro. Dichos lugares fueron escenarios para la presentación de las muestras de Cine Iberoamericano y tuvieron una gran concentración de espectadores a lo largo de su semana de duración. Esta encuesta fue aplicada durante una semana, iniciando el domingo 14 y finalizando el sábado 20 de noviembre del 2010.

Para calcular los efectos indirectos, era necesario acercarnos a la previsión de una serie de datos, los cuales fueron generados a partir de las respuestas de los espectadores encuestados. Estos datos son necesarios para conocer el gasto generado por los asistentes al Festival, es decir, sus efectos indirectos.

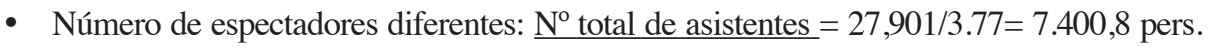
$\mathrm{N}^{\circ}$ medio de películas por espectador

- Origen de los espectadores:

\section{Cuadro 4}

\section{ORIGEN DE LOS ESPECTADORES}

\begin{tabular}{|c|c|c|}
\hline Origen & Personas & Porcentaje \\
\hline Locales & $5.136,16$ & $69.4 \%$ \\
\hline No locales & $2.264,64$ & $30.6 \%$ \\
\hline TOTAL & $\mathbf{7 . 4 0 0 , 8}$ & $\mathbf{1 0 0 \%}$ \\
\hline
\end{tabular}

Fuente: Elaboración propia.

- Motivo de asistencia:

De ese $30 \%(2.264,64)$ de espectadores que acudieron al festival procedentes de otros municipios diferentes al onubense, el 49,3\%, lo que representa unas 1.116,46 personas, tenían como principal motivo del viaje a Huelva la asistencia al propio festival del cine Iberoamericano, el $29.3 \%$, unas 663.54 personas, consideraron que esta asistencia estaba entre uno de sus motivos para desplazarse a la capital y para el $18.1 \%$ el festival no influyó en la decisión de su viaje. Un 3.3\% que no contestó.

De estas poblaciones, para el análisis de los efectos económicos indirectos, solo se considerarán las dos primeras. De tal forma, que lo gastado por los asistentes que acudieron a Huelva principalmente para la asistencia al Festival se computará en un 100\%, mientras que el gasto realizado por los asistentes que viajaron a Huelva motivados, en parte, por el Festival, se considerará en un 50\%. El gasto realizado por el resto de asistentes no computará a la hora de calcular este efecto. 


\section{Cuadro 5}

MOTIVOS DE ASISTENCIA A HUELVA

\begin{tabular}{|l|c|c|}
\hline \multicolumn{1}{|c|}{ Motivos asistencia a Huelva } & Personas & Porcentaje \\
\hline Principal: el Festival & 1116,46 & 49,3 \\
\hline Uno de los motivos & 663,54 & 29,3 \\
\hline No ha influido el Festival & 409,90 & 18,1 \\
\hline Ns/Nc & 74,73 & 3,3 \\
\hline Total & $2.264,64$ & $\mathbf{1 0 0 , 0}$ \\
\hline
\end{tabular}

Fuente: Elaboración propia.

- Gasto medio diario por partidas:

En el Cuadro 6, fruto de la encuesta realizada a los asistentes, se recoge el gasto medio diario que realizaron estos en: alojamiento, comidas entretenimiento, transporte, golosinas y otras compras (sin objetos del festival ni entradas).

\section{Cuadro 6}

\section{GASTOS MEDIOS POR PARTIDAS (€)}

\begin{tabular}{|l|c|c|c|c|c|c|c|}
\hline & Alojamiento & Comida & $\begin{array}{c}\text { Entreteni- } \\
\text { miento: café, } \\
\text { copas, etc. }\end{array}$ & $\begin{array}{c}\text { Transporte } \\
\text { en la } \\
\text { ciudad }\end{array}$ & $\begin{array}{c}\text { Caramelos } \\
\text { Frutos secos } \\
\text { Etc. }\end{array}$ & $\begin{array}{c}\text { Otras } \\
\text { compras } \\
\text { (sin objetos } \\
\text { festival ni } \\
\text { entradas) }\end{array}$ & Otros \\
\hline Media & 2,61 & 2,93 & 3,25 & 2,88 & 2,94 & 3 & 5,37 \\
\hline Marca de clase & 7,42 & 13 & 6,33 & 4,7 & 4,09 & 6,5 & 11,1 \\
\hline Total euros & 19.37 & 38.09 & 20,6 & 13,54 & 12,05 & 19,52 & 59,6 \\
\hline
\end{tabular}

Fuente: Elaboración propia.

- Número medios de días y noches de estancia en Huelva: Días= 4 ; Noches $=5.11$

\section{Cuadro 7}

\section{GASTOS DE LOS ASISTENTES NO LOCALES (€)}

\begin{tabular}{|l|c|c|c|c|}
\hline \multirow{2}{*}{} & \multicolumn{2}{|c|}{ Motivo principal } & \multicolumn{2}{c|}{ Motivo secundario } \\
\cline { 2 - 5 } & $\begin{array}{c}\text { Gasto medio } \\
\text { diario }^{\mathbf{2}}\end{array}$ & $\begin{array}{c}\text { Gasto estancia } \\
\mathbf{( 1 0 0 \% )}\end{array}$ & $\begin{array}{c}\text { Gasto medio } \\
\text { diario }\end{array}$ & $\begin{array}{c}\text { Gasto estancia } \\
\mathbf{( 5 0 \% )}\end{array}$ \\
\hline Alojamiento & 19,37 & 96,87 & $(11,81)$ & $(29,53)$ \\
\hline Comidas & 38,09 & 150,4 & $(12,76)$ & $(25,52)$ \\
\hline Entretenimiento & 20,6 & 82,36 & $(5,48)$ & $(10,96)$ \\
\hline Transporte & 6,7 & 26,81 & $(3,47)$ & $(6,94)$ \\
\hline Otras Compras & 19,52 & 78,09 & $(9,5)$ & $(19)$ \\
\hline Gasto de estancia por persona & & 434,53 & & $(91,95)$ \\
\hline $\mathrm{N}^{\text {o de espectadores }}$ & & $2.264,64(49 \%)$ & & $2.264,64(29 \%)$ \\
\hline GASTO TOTAL & & $\mathbf{4 8 2 . 1 8 6 , 4 7}$ & & $\mathbf{6 0 . 3 8 7 , 7 6}$ \\
\hline
\end{tabular}

Fuente: Elaboración propia. 
Como puede apreciarse, las partidas más destacadas son la de alojamiento y comidas, las cuales alcanzan entre ambas casi un $57 \%$ del gasto medio total por persona, seguido por el gasto en entretenimiento que ocupa un $19 \%$ del total. El gasto total de las personas que asistieron al festival Iberoamericano de Huelva, es decir, el efecto económico indirecto es de $542.574,23$.

En el cuadro 8 se recoge la distribución entre las diferentes partidas de ese efecto económico indirecto total. En este sentido, se constata que un 57,23\% del gasto de los asistentes a la ciudad de Huelva, motivados, de una u otra forma, por el Festival de Cine Iberoamericano, lo realizan en el sector de la hostelería, seguido de las compras y del entretenimiento con un $18 \%$ respectivamente.

De esta forma, como ya se apuntaba en el apartado anterior, referente al análisis de los trabajos de investigación previamente realizados, el sector de la hostelería (hotelería y restauración) y del comercio al por menor, motivado por las compras, son los que, en mayor medida, salen beneficiados por la organización de este festival de cine, fundamentalmente, por los gastos que realizan los que visitan la ciudad, los cuales representa alrededor del $30 \%$ del público total.

\section{Cuadro 8 \\ DISTRIBUCIÓN DEL GASTO DE LOS ASISTENTES NO LOCALES (€)}

\begin{tabular}{|l|c|c|}
\hline \multicolumn{1}{|c|}{ Partidas } & Gasto Total & Porcentaje \\
\hline Alojamiento & $126.887,56$ & 23,38 \\
\hline Comidas & $183.654,63$ & 33,85 \\
\hline Entretenimiento & $98.590,4$ & 18,17 \\
\hline Transportes & $34.308,1$ & 6,32 \\
\hline Compras & $99.132,38$ & 18,27 \\
\hline TOTAL & $\mathbf{5 4 2 . 5 7 4 , 2 3}$ & $\mathbf{1 0 0 , 0 0}$ \\
\hline
\end{tabular}

Fuente: Elaboración propia.

Por tanto, podría decirse que el esfuerzo inversor que se realiza a la hora de organizar el Festival de Cine Iberoamericano de Huelva, en primera instancia, sin entrar en el análisis de los efectos económicos inducidos que tiene en la economía local, casi queda compensado con los gastos y el consumo que generan en la cuidad los asistentes al mismo. De forma que, mientras el presupuesto y, por tanto, la cantidad gastada o, mejor dicho, invertida, en la 36 Edición del Festival fue de 682.067 euros, lo que los asistentes consumieron fue de 542.574,23 euros, lo cual nos lleva a concluir que sólo con los efectos económicos indirectos del consumo de los asistentes, en aspectos tales como hotelería, restauración y entretenimiento, casi se recupera la inversión que se realiza a la hora de organizar este evento para la ciudad.

No obstante, en el siguiente apartado se calcula el efecto inducido, es decir, el efecto arrastre o multiplicador, que ejercen todos estos gastos, tanto directos como indirectos, en la economía de la ciudad. Este efecto inducido se evidencia en una puesta en funcionamiento y una mayor producción de otros sectores económicos, diferentes a la hostelería o el comercio, sobre los cuales, tal y como hemos comentado, recae los efectos indirectos. 


\section{Efectos inducidos}

Conocidos los gastos directos e indirectos, podemos determinar el efecto económico inducido del Festival de Cine Iberoamericano a través de los multiplicadores interiores sectoriales de la región andaluza para el año 20056. A partir del desglose de los gastos totales, la matriz de coeficientes técnicos regionales permite estimar el esfuerzo de producción que tiene que realizar la economía onubense para atender la demanda de bienes y servicios existente en la ciudad, motivados por la celebración del Festival, ya sea a partir de los gastos que genera la propia organización del Festival o de los que lleva a cabo los asistentes al mismo. Estos efectos inducidos totales se recogen en el Cuadro 9.

\section{Cuadro 9 \\ EFECTOS TOTALES INDUCIDOS SOBRE LA ECONOMÍA ONUBENSE (€)}

\begin{tabular}{|l|c|c|c|c|c|}
\hline & $\begin{array}{c}\text { Gastos } \\
\text { directos }\end{array}$ & $\begin{array}{c}\text { Gastos } \\
\text { indirectos }\end{array}$ & Gasto total & Multiplicador & $\begin{array}{c}\text { Efecto } \\
\text { inducido } \\
\text { Total }\end{array}$ \\
\hline Personal & 82.674 & - & 82.674 & 1,53 & $126.491,22$ \\
\hline $\begin{array}{l}\text { Alojamiento y } \\
\text { restauración }\end{array}$ & 109.876 & 310542,19 & $420.418,19$ & 1,31 & $550.747,83$ \\
\hline Transporte & & 34.308 & 34.308 & 1,90 & $65.185,2$ \\
\hline Compras al por menor & - & $99.132,38$ & $99.132,38$ & 1,04 & $103.097,68$ \\
\hline Entretenimiento & & $98.590,4$ & $98.590,4$ & 1,28 & $126.195,71$ \\
\hline Programa & 153.626 & - & 153.626 & 1,41 & $216.612,66$ \\
\hline $\begin{array}{l}\text { Comunicación y } \\
\text { publicidad }\end{array}$ & 79.599 & - & 79.599 & 1,16 & $92.334,84$ \\
\hline Administración & 159.973 & & 159.973 & 1,63 & $260.755,99$ \\
\hline TOTALES & $\mathbf{6 2 0 . 0 5 6}$ & $\mathbf{5 0 8 . 2 6 4 , 9 7}$ & $\mathbf{1 . 1 2 8 . 3 2 0 , 9 7}$ & $\mathbf{1 , 3 6}$ & $\mathbf{1 . 5 4 1 . 4 2 1 , \mathbf { 1 3 }}$ \\
\hline
\end{tabular}

Fuente: Elaboración propia

Por tanto, la repercusión o gasto total del Festival sobre la economía de la ciudad de Huelva sería la suma de los efectos directos, indirectos e inducidos, dando lugar al efecto inducido total, recogido en la última columna del Cuadro anterior. En este sentido, podemos decir que el Festival de Cine Iberoamericano genera un total de 1,54 millones de Euros que se diluyen por el tejido empresarial onubense. De tal forma, que podría decirse que el presupuesto que tiene asignado la organización del Festival (682.067 € para el 2010) representa un $44,2 \%$ del total del flujo financiero que mueve el mismo a lo largo de todo el año en la ciudad de Huelva.

6 Estos multiplicadores utilizados hacen referencia a la economía andaluza, y no a la ciudad de Huelva, por lo que existe un desfase en los efectos inducidos y, por tanto, en el efecto total, como un máximo. No obstante, consideramos que los efectos del Festival de Cine Iberoamericano de Huelva se quedan principalmente en el tejido productivo de la ciudad por las características de los sectores de actividad afectados, los cuales, en su mayor parte, se localizan en la propia ciudad. Estos sectores a los que nos referimos son: hotelería, restauración, compra al por menor, transporte, etc. 
Por consiguiente, concluimos que, al igual que se ha podido comprobar en otros trabajos de investigación de este tipo, que han utilizado la misma metodología, en lo que a los efectos económicos respecta, es decir, considerando sólo los aspectos cuantitativos, el Festival de Cine Iberoamericano representa una verdadera inversión económica para la ciudad, al generar un flujo financiero cifrado en más del doble respecto a esa inversión inicial que se realiza con la organización del mismo.

Los sectores más beneficiados por la celebración del Festival, tal y como se extrae del Cuadro 9, son los de la hostelería y la restauración, es decir, los sectores más relacionados con el sector turístico. No obstante, también hay otros sectores que se ven muy beneficiados por la celebración del Festival, como son: los servicios de edición e imprenta y el comercio al por menor de la ciudad.

Por consiguiente, podría concluirse que la organización de este festival, sin entrar a considerar otros posibles efectos, de corte más cualitativo, que pudiera tener sobre el desarrollo local de la ciudad (mejora del nivel cultural, ampliación de las infraestructuras y oferta cultural, desestacionalización del turismo, promoción del destino turístico, etc.), se presenta como una importante actuación estratégica de cara a impulsar su desarrollo económico local, al generar unos ingresos muy superiores a los gastos; ingresos que se quedan, en su mayor parte, en el tejido productivo local.

\section{CONCLUSIONES}

Dentro del apartado de las conclusiones, dividiremos éstas en base a los dos grandes bloques que se pueden identificar en esta investigación. En este sentido, haremos referencia a las principales conclusiones del análisis bibliográfico y marco teórico para, posteriormente, comentar las principales conclusiones a las que se ha llegado en el análisis empírico de caso, es decir, las relacionadas con el impacto económico local que genera la organización del Festival de Cine Iberoamericano en la ciudad de Huelva.

Respecto a la revisión bibliográfica realizada cabe concluir que:

- Los estudios consultados coinciden que en que la organización de estos eventos, relacionados con la cultura, sobre todo en lo que al cine respecta, genera importantes impactos, tanto sociales como económicos, para el territorio donde se organizan.

- Respecto a los impactos económicos, los ingresos suelen superar a los gastos que ocasiona la organización de los mismos, ya sea en forma de efectos económicos directos, indirectos o inducidos.

- Respecto al impacto social, la organización de estos eventos contribuye a mejorar la calidad de vida y el nivel cultural de gran parte de la población local que habita en estos territorios.

- Por otro lado, este tipo de eventos culturales representan un importante atractivo para promocionar turísticamente los territorios (destinos turísticos) y configurar productos turísticos relacionados con el turismo cultural, y, para el caso del cine, con el turismo cinematográfico. En este sentido, no debe olvidarse que estas tipologías de turismos, en las últimas décadas, comienzan a crecer a tasas superiores a lo que lo hace la media del sector, siendo, al mismo tiempo, una tipología que 
contribuye a solucionar, en parte, los problemas de estacionalidad del sector, la necesidad de elevar sus niveles de competencia, etc.

- Además, el tipo de turista que participa de estos eventos, entre otras características, suele tener un nivel formativo alto, unos ingresos medios-altos y suele repetir en sus visitas, lo cual representa un segmento de la demanda turística bastante atractivo para los destinos.

En lo que respecta al análisis de caso, podemos concluir que el Festival de Cine Iberoamericano, en el año 2010, se estima que generó alrededor de 1,54 millones de Euros, que se diluyen por el tejido empresarial onubense. De tal forma, que podría decirse que el presupuesto que tenía asignado la organización del Festival (682.067 € para el 2010) representa un $44,2 \%$ del total del flujo financiero que mueve el mismo en la ciudad de Huelva.

Por consiguiente, se puede concluir que, ratificando las conclusiones a las que se llega en otros estudios, utilizando esta misma metodología, en lo que a los efectos económicos respecta, el Festival de Cine Iberoamericano representa una verdadera inversión para la ciudad, al generar un volumen de flujo financiero estimado en más del doble respecto a la inversión inicial que se realiza con la organización del mismo.

Los sectores más beneficiados por la celebración del Festival son los de la hostelería y restauración, es decir, los sectores más relacionados con el sector turístico. No obstante, también hay otros sectores que se ven muy beneficiados por la celebración del Festival, como son: los servicios de edición e imprenta y el comercio al por menor de la ciudad.

En definitiva, desde un punto de vista económico y cuantitativo, queda demostrado, al igual que concluyen otros muchos estudios consultados, que, para el desarrollo económico local de la ciudad de Huelva, la organización y celebración del Festival de Cine Iberoamericano genera un impacto económico muy positivo, configurándose, desde este punto de vista, en una actuación estratégica a tener en cuenta para ello.

No obstante, en futuros trabajos de investigación se deberá abordar el impacto que ejerce la organización de este festival en aspectos tales como el turismo en la ciudad, su cultura, la población local, etc., profundizando, por tanto, en aspectos de corte cualitativos, necesarios de considerar a la hora de dinamizar procesos de desarrollo local.

\section{BIBLIOGRAFÍA}

ASUAGA, C. et al (2011): «Análisis preliminar del impacto económico de la temporada de ópera 2010 del Teatro Solís», I Jornada Académica de la Facultad de Ciencias Económicas y de Administración, Universidad de la República, Uruguay.

BARROSO GONZÁLEZ, M.O. y FLORES RUIZ, D. (2006): «La competitividad internacional de destinos turísticos: del enfoque macroeconómico al enfoque estratégico», Cuadernos de Turismo, $\mathrm{n}^{\circ} 17$, pp. 7-24.

BEETON, S. (2005): Film-Induced Tourism, Bristol, Channel View Publications.

CAPAUL, M. (1988): «El impacto económico del festival de cine de San Sebastián», Estudios Empresariales, nº 67, pp. 47-54. 
CONSEJERÍA DE AGRICULTURA Y PESCA (2003a): Resultados de la Iniciativa Comunitaria LEADER II, Sevilla, Consejería de Agricultura y Pesca, Empresa Pública Desarrollo Agrario y Pesquero.

CONSEJERÍA DE AGRICULTURA Y PESCA (2003b): Resultados del Programa Operativo PRODER, Sevilla, Consejería de Agricultura y Pesca, Empresa Pública Desarrollo Agrario y Pesquero.

DEL REY-REGUILLO, A. (2007): «Introducción», en Cine, Imaginario y Turismo, Valencia, Tirant lo Blanch, pp. 147-175.

DEVESA, M. et al (2012): «Repercusiones económicas y sociales de los festivales culturales: el caso del Festival Internacional de Cine de Valdivia», CEURE, VOL 38, $\mathrm{n}^{\circ}$ 115 pp. 95-115.

DEVESA, M. (2006): El impacto económico de los festivales culturales. El caso de la Semana Internacional de Cine de Valladolid, Madrid, Fundación Autor.

DEVESA, M. y HERRERO, L.C. (2008): «El atractivo turístico de los festivales culturales y su incidencia en el desarrollo local», XI Congreso de Turismo Universidad y Empresa, Castellón, España.

DOMÍNGUEZ AZCUE, J. (2014): Análisis de la imagen de destino a través de la cinematografía: Málaga, Tesina, Universidad de Málaga.

ELIZAGARATE, V. (2008): Marketing de ciudades. Estrategias para el desarrollo de ciudades atractivas y competitivas en un mundo global, Madrid, Pirámides.

ESPINAL, N.E. (2006): «Economía de la cultura», Ensayos de Economía, Octubre 2006, pp. 71-82.

EVANS, M. (1997): Plugging into TV tourism, Insights, $\mathrm{n}^{\circ} 3$, pp. 35-38.

FLORES RUIZ, D. y BARROSO GONZÁLEZ, M.O. (2012): «El turismo rural como estrategia de desarrollo rural sostenible. Los parques naturales andaluces», Revista de Estudios Empresariales. Segunda Época, 1 (2012), p.p. 59-83.

FREY, B.S. (1994): «The economics of Music Festivals», Journal of Cultural Economics: $\mathrm{n}^{\circ} 18$ (1): pp. 29-39.

GÁMIR, A. y VALDÉS, C.M. (2007): «Cine y geografía: espacio geográfico, paisaje y territorio en las producciones cinematográficas», Boletín de la Asociación de Geógrafo Españoles, n 45 , pp. 157-190.

GARCÍA, A. y ALBURQUERQUE, F.J. (2003): El turismo cultural y el de sol y playa: ¿sustitutivos o complementarios?, Cuadernos de turismo, no 11 pp. 97-105.

GARCÍA, M.I et al (2003): «The Economic Dimensión of the Cultural and Leisure Industry in Spain», Journal of Cultural Economics, n ${ }^{\circ} 27$, pp. 9-30.

GETZ, D. (1991): Festivals, Special Events ant Toursism, Nueva York, Van Nostrand Reinhold.

GILBSON, C et al (2010): «Cultural Festivals and Economic Development in Nonmetropolitan Australia». Journal of Planning Educations and Research. Vol. 29 n 3.

GRANDE, J. (2001): «Análisis de la oferta de turismo cultural en España», Estudios Turísticos, $\mathrm{n}^{\mathrm{o}} 150$, pp. 15-40.

GUGGENHEIN BILBAO (2003): Impacto económico de las actividades del museo Guggenhein Bilbao en la economía del País Vasco en el año 2002, Bilbao, Mimeo. 
HERRERO, L.C. et al (2006): «The Economic Impact of Cultural Events: a Case-Study of Salamanca 2002, European capital of culture», European Urban and Regional Studies, http://eur.sagepub.com/content/13/1/41

HERRERO, L.C. et al (2011): «Measuring the economic value and social viability of a cultural festival as a tourism prototype», Tourism Economic: the business and finance of tourism and recreation, vol. 17, $\mathrm{n}^{\circ} 3$, pp. 639-653.

HUDSON, S. y BERENT RITCHIE, J.R. (2006): «Promoting Destinations Via Film Tourism: An Empirical Identification of Supporting Marketing Iniciatives», Journal of travel research $\mathrm{n}^{\circ} 44$, pp. 387-396.

HUDSON, S. y BRENT, J.B. (2006): «Film Tourism and Destination Marketing: The Case of Captain Corelli's Mandolin», Journal of Vacation Marketing, $\mathrm{n}^{\circ}$ 12, pp. 256-268.

MARTÍNEZ PUCHE, A. (2008): «El cine como soporte didáctico para explicar la evolución del viaje y la actividad turística», $\mathrm{n}^{\circ}$ 22, pp. 145-163.

McKERCHER, B y CROSS, H. (2002): Cultural Tourism: the Partnership between Tourism and Cultural Heritage, EEUU, The Haworth Press.

O’HAGAN, J. (1989): The economic and social contribution of the Wexford Opera Festival, Dublín,Trinity College.

PERLES, J.F. (2006): «Análisis del impacto económico de eventos: una aplicación a fiestas populares de proyección turística», Cuadernos de Turismo, nº 17 pp.147-166.

RICHARDS, G. (1996): Cultural Tourism in Europe, Wallingford, CABI.

RODRÍGUEZ, A. (2002): «Reinventar la ciudad: milagros y espejismos de la revitalización urbana de Bilbao», Lan Harremanak/6, I, pp. 69-108.

RODRÍGUEZ, M.L. et al (2013): «Nuevos segmentos turístico culturales. Una aproximación al comportamiento del consumidor turístico cinematográfico», Cuadernos de Turismo, $\mathrm{n}^{\mathrm{o}} 32$, pp. 259-279.

RODRIGUEZ CAMPOS, M.L. y FRAIZ BREA, J. (2010): «Consideraciones estratégicas para la promoción del turismo en Galicia a través del cine», Revista Gallega de Economía, vol. 19, $\mathrm{n}^{\circ}$ 2, pp. 1-10, Vigo.

RODRÍGUEZ CAMPOS, M.L. y FRAIZ BREA, J. (2011): «La imagen turística en el cine: evaluación del impacto de un largometraje en la imagen percibida del destino en el que se emplaza», Revista InterXesiones, $\mathrm{n}^{\circ} 2$ pp. 39-53.

ROESCH (2009): The Experiences of Film Location Tourists, Clevedon, Channel View Publications.

SANDOVAL MARTÍN (1998): «Promoción turística a través del sector audiovisual. El caso de Canarias». Revista Latina de Comunicación Social, $\mathrm{n}^{\circ}$ 9, Recuperado el 6 de diciembre de 2014 de: http://www.ull.es/publicaciones/latina/a/37san.htm

SCOTTISH TOURIST BOARD (1996): Edinburgh Festivals Economics Study, Mimeo, Edinburgh.

SHELLARD, D (2007): Economic impact study of UK theatre. Arts Council. England. THROSBY, D. (2001): Economía y Cultura, Cambridge University Press, Cambridge.

TYRREL, B.J. e ISMAIL, J.A. (2005): «A methodology for estimating the attendance and economic impact of an open-gate festival». Event management, n ${ }^{\circ} 9$ pp. 111-118. YEOMAN, I (2004): Festival and events management, Oxford, Elsevier. 